\title{
ZERO SETS OF ENTIRE FUNCTIONS OF EXPONENTIAL TYPE WITH ADDITIONAL CONDITIONS ON THE REAL AXIS
}

\author{
S. YU. FAVOROV
}

\begin{abstract}
A complete description of the zero sets is obtained for some well-known classes of entire functions of exponential growth.
\end{abstract}

We denote by $B$ the class of entire functions of exponential type bounded on the real axis; by $C$, the class of Cartwright's entire functions, i.e., entire functions $f$ of exponential type with the property

$$
\int_{-\infty}^{\infty} \frac{\log ^{+}|f(x)|}{1+x^{2}} d x<\infty
$$

and by $D$, the class of entire functions $f$ of exponential type with the following property: for each sequence $h_{m} \in \mathbb{R}$ there exists a subsequence $h_{m}^{\prime}$ and a function $\tilde{f} \not \equiv 0$ such that the sequence $f\left(z+h_{m}^{\prime}\right)$ converges uniformly to a function $\tilde{f}$ on every compact subset of $\mathbb{C}$.

It is easily seen that $D \subset B \subset C$. The classes $B$ and $C$ are well known and play an important role in various topics of complex and functional analysis (see [1, 2, 3]); the class $D$ is an extension of the well-known class of sine-type functions (see [2]), and it inherits some properties of sine-type functions.

Here we consider sequences $\left\{a_{k}\right\}$ of complex numbers without finite limit points; note that each $\left\{a_{k}\right\}$ may appear in the sequence with a finite multiplicity. We say that a sequence $\left\{a_{k}\right\}$ is the zero set for an entire function $f$ if $f(a) \neq 0$ for $a \notin\left\{a_{k}\right\}$ and $f(a)=f^{\prime}(a)=\cdots=f^{(p-1)}(a)=0, f^{(p)}(a) \neq 0$, where $p$ is the multiplicity of $a$ in $\left\{a_{k}\right\}$.

The zero set of any function $f \in C$ satisfies the following conditions:

and

$$
\begin{aligned}
& \sum_{a_{k} \neq 0}\left|\operatorname{Im} a_{k}^{-1}\right|<\infty ; \\
& \text { the limit } \lim _{R \rightarrow \infty} \sum_{0<\left|a_{k}\right|<R} a_{k}^{-1} \text { exists; }
\end{aligned}
$$

for every $0<\alpha \leq \pi / 2$, where $d$ is the width of the indicator diagram of $f$ (see [2, p. 127]). Thus, every function $f \in C$ has the form

$$
\begin{gathered}
\lim _{R \rightarrow \infty} R^{-1} \operatorname{card}\left\{a_{k}: 0<\left|a_{k}\right|<R,\left|\arg a_{k}\right| \leq \alpha\right\}=d / 2 \pi, \\
\lim _{R \rightarrow \infty} R^{-1} \operatorname{card}\left\{a_{k}: 0<\left|a_{k}\right|<R,\left|\arg a_{k}-\pi\right| \leq \alpha\right\}=d / 2 \pi,
\end{gathered}
$$

$$
f(z)=A z^{s} e^{i \lambda z} \lim _{R \rightarrow \infty} \prod_{0<\left|a_{k}\right|<R}\left(1-z / a_{k}\right)
$$

2000 Mathematics Subject Classification. Primary 30D15, Secondary 30D10, 30D45.

Key words and phrases. Entire function of exponential type, Cartwright's function, zero set. 
with $A \in \mathbb{C}, \lambda \in \mathbb{R}$ (see [2, p. 130]). However, there are zero sets satisfying (2)-(5) and such that no entire function with this zero set belongs to $C$.1

In [1, Appendix VI], B. Levin investigated zero sets $\left\{a_{k}\right\}, k \in \mathbb{Z}$, for functions of class $B$ with the additional property $\sup _{k}\left|a_{k}-h k\right|<\infty$ for some $h \in \mathbb{R}$. In the present paper we obtain a complete description of the zero sets for the classes $B, C$, and $D$.

In what follows, we put $n(c, t)=\operatorname{card}\left\{a_{k}:\left|a_{k}-c\right| \leq t\right\}$ for $c \in \mathbb{C}$. Assume also that the sequence $\left\{a_{k}\right\}$ satisfies the conditions

$$
n(0, t)=O(t), \quad t \rightarrow \infty
$$

and

$$
n(0, t+1)-n(0, t)=o(t), \quad t \rightarrow \infty .
$$

It is clear that (7) and (8) are preserved if we replace $n(0, t)$ with $n(c, t)$.

Theorem 1. A sequence $\left\{a_{k}\right\} \subset \mathbb{C}$ is the zero set of some function $f \in C$ if and only if it satisfies conditions (3), (7), and (8) and

$$
\int_{-\infty}^{\infty}\left[\int_{0}^{\infty}[n(b, t)-n(x, t)] t^{-1} d t\right]^{+} \frac{d x}{1+x^{2}}<\infty
$$

for some point $b \in \mathbb{R} \backslash\left\{a_{k}\right\}$.

Theorem 2. A sequence $\left\{a_{k}\right\} \subset \mathbb{C}$ is the zero set of some function $f \in B$ if and only if it satisfies conditions (3), (7), and (8), and

$$
\sup _{x \in \mathbb{R}} \int_{0}^{\infty}[n(b, t)-n(x, t)] t^{-1} d t<\infty
$$

for some point $b \in \mathbb{R} \backslash\left\{a_{k}\right\}$.2

Theorem 3. A sequence $\left\{a_{k}\right\} \subset \mathbb{C}$ is the zero set of some function $f \in D$ if and only if it satisfies conditions (3), (7), and (8), and

$$
\sup _{x \in \mathbb{R}}\left|\int_{1}^{\infty}[n(0, t)-n(x, t)] t^{-1} d t\right|<\infty .
$$

The proofs of Theorems 1-3 are based on the following lemma.

Lemma 1. Suppose a sequence $\left\{a_{k}\right\} \subset \mathbb{C} \backslash\{0\}$ satisfies (3), (7), and (8). Then

$$
g(z)=\lim _{R \rightarrow \infty} \prod_{\left|a_{k}\right|<R}\left(1-z / a_{k}\right)
$$

is a well-defined entire function of a finite exponential type, and

$$
\log |g(z)|=\int_{0}^{\infty}[n(0, t)-n(z, t)] t^{-1} d t
$$

for all $z \in \mathbb{C}$. Moreover, if $z_{0}$ is a point of the sequence $\left\{a_{k}\right\}$ with multiplicity $l=$ $n\left(z_{0}, 0\right)>0$, then

$$
\log \left|\frac{g^{(l)}\left(z_{0}\right)}{l !}\right|=\int_{1}^{\infty} \frac{n(0, t)-n\left(z_{0}, t\right)}{t} d t+\int_{0}^{1} \frac{n(0, t)-n\left(z_{0}, t\right)+l}{t} d t .
$$

\footnotetext{
${ }^{1}$ For example, take $a_{1}=-e^{2}$ and consider reals $a_{k}<a_{1}, k=2, \ldots$, such that the number $n(r)=$ $\operatorname{card}\left\{a_{k}: a_{k} \geq-r\right\}$ is the integral part of $r / \log ^{2} r$ for each $r \geq e^{2}$. Then (2)-(5) are fulfilled and the function (6) with $s=0$ does not satisfy (1) because for $x>e^{2}$ we have

$$
\log |f(x)|=\int_{1}^{\infty} \log (1+x / t) d n(t)=\int_{1}^{\infty} \frac{x n(t) d t}{t(x+t)}>1+\int_{x}^{\infty} \frac{x d t}{2 t \log ^{2} t}=1+\frac{x}{2 \log x}
$$

(this idea belongs to Professor A. Grishin).

${ }^{2}$ Theorems 1 and 2 were announced (with my permission) in the survey 4 p. 45].
} 
Proof of Lemma 1. Relation (77) implies that the integral $\int_{0}^{\infty} n(0, t) t^{-3} d t$ is finite; hence,

$$
\sum\left|a_{k}\right|^{-2}=\int_{0}^{\infty} t^{-2} d n(0, t)<\infty .
$$

Let $K$ be a fixed disk in the complex plane. Then

$$
\sum_{R<\left|a_{k}\right|<R^{\prime}} \log \left(1-\frac{z}{a_{k}}\right)-\sum_{R<\left|a_{k}\right|<R^{\prime}} \frac{z}{a_{k}} \rightarrow 0
$$

as $R, R^{\prime} \rightarrow \infty$ uniformly in $z \in K$. Hence, uniformly in $z \in K$ we have

$$
\lim _{R \rightarrow \infty} \sum_{\left|a_{k}\right|>R} \log \left(1-\frac{z}{a_{k}}\right)=0
$$

and the function $g$ is well defined. Now, conditions (3) and (7) show that the function (12) is of a finite exponential type.

Note that (13) is trivial for $z=a_{k}$. Fix $z \notin\left\{a_{k}\right\}$. Suppose $\varepsilon>0$ is such that $n(0, \varepsilon)=n(z, \varepsilon)=0$. Then for any $R<\infty$ we have

$$
\begin{aligned}
\sum_{\left|a_{k}\right| \leq R} & \left(\log \left|z-a_{k}\right|-\log \left|a_{k}\right|\right) \\
= & \sum_{\varepsilon \leq\left|a_{k}-z\right| \leq R} \log \left|z-a_{k}\right|-\sum_{\varepsilon \leq\left|a_{k}\right| \leq R} \log \left|a_{k}\right| \\
& +\sum_{\left|a_{k}\right| \leq R,\left|z-a_{k}\right|>R} \log \left|z-a_{k}\right|-\sum_{\left|z-a_{k}\right| \leq R,\left|a_{k}\right|>R} \log \left|z-a_{k}\right| .
\end{aligned}
$$

Furthermore,

$$
\sum_{\varepsilon \leq\left|a_{k}-z\right| \leq R} \log \left|z-a_{k}\right|=n(z, R) \log R-\int_{\varepsilon}^{R} n(z, t) t^{-1} d t
$$

and

$$
\begin{aligned}
(\log R) & {[n(z, R)-n(0, R)]+\sum_{\left|a_{k}\right| \leq R,\left|z-a_{k}\right|>R} \log \left|z-a_{k}\right| } \\
& -\sum_{\left|z-a_{k}\right| \leq R,\left|a_{k}\right|>R} \log \left|z-a_{k}\right| \\
= & \sum_{\left|a_{k}\right| \leq R,\left|z-a_{k}\right|>R} \log \frac{\left|z-a_{k}\right|}{R}+\sum_{\left|z-a_{k}\right| \leq R,\left|a_{k}\right|>R} \log \frac{R}{\left|z-a_{k}\right|} .
\end{aligned}
$$

Observe that if $\left|z-a_{k}\right|>R$ and $\left|a_{k}\right| \leq R$, then $\left|a_{k}\right|>R-|z|$ and $\left|z-a_{k}\right| \leq|z|+R$. Also, if $\left|a_{k}\right|>R$ and $\left|z-a_{k}\right| \leq R$, then $\left|a_{k}\right| \leq R+|z|$ and $\left|z-a_{k}\right|>R-|z|$. Hence, as $R \rightarrow \infty$, the right-hand side of (18) does not exceed the quantity

$$
O(|z| / R)[n(0, R)-n(0, R-|z|)]+O(|z| / R)[n(0, R+|z|)-n(0, R)] .
$$

Recalling (8), we see that (19) tends to zero for fixed $z$ as $R \rightarrow \infty$. Therefore, (13) follows from (17) and (16). In order to get (14), it suffices to apply (13) to the function $g(z)\left(z-z_{0}\right)^{-l} z_{0}^{l}$. Lemma 1 is proved.

Proof of Theorems 1-3. Relation (15) shows that the limits as $R \rightarrow \infty$ of the sums $\sum_{\left|a_{k}-c\right|<R}\left(a_{k}-c\right)^{-1}$ and $\sum_{\left|a_{k}-c\right|<R}\left(a_{k}\right)^{-1}$ exist simultaneously; the latter sum differs from $\sum_{\left|a_{k}\right|<R}\left(a_{k}\right)^{-1}$ in no more than $n(0, R+|c|)-n(0, R-|c|)=o(R)$ terms, and the modulus of each of these terms does not exceed $(R-|c|)^{-1}$. Therefore, (3) is invariant with respect to the shift of the origin. Applying Lemma 1 to the function 
$\lim _{R \rightarrow \infty} \prod_{\left|a_{k}-c\right|<R}\left(1-z /\left(a_{k}-c\right)\right)$, we see that $\left[\int_{0}^{\infty}[n(c, t)-n(x, t)] t^{-1} d t\right]^{+}=O(|x|)$ as $|x| \rightarrow \infty$. Therefore, (9) is also invariant with respect to the shift of the real parameter $b$. Hence, there is no loss of generality in assuming that $0 \notin\left\{a_{k}\right\}$ and $b=0$ in (9) and (10).

Lemma 1 immediately shows that, under the hypotheses of Theorems 1 or 2 , the function (12) belongs to $C$ or to $B$, respectively. Let $\left\{a_{k}\right\}$ satisfy the hypothesis of Theorem 3. Using (13), (14), and the Jensen formula

$$
\frac{1}{2 \pi} \int_{0}^{2 \pi} \log \left|g\left(z+e^{i \theta}\right)\right| d \theta=\log \left|\frac{g^{(l)}(z)}{l !}\right|+\int_{0}^{1} \frac{n(z, t)-n(z, 0)}{t} d t
$$

with $l=n(z, 0)$, we get

$$
\frac{1}{2 \pi} \int_{0}^{2 \pi} \log \left|g\left(z+e^{i \theta}\right)\right| d \theta=\int_{1}^{\infty} \frac{n(0, t)-n(z, t)}{t} d t+\int_{0}^{1} \frac{n(0, t)}{t} d t .
$$

Hence, (11) and the inequality

$$
\log |g(z)| \leq \frac{1}{2 \pi} \int_{0}^{2 \pi} \log \left|g\left(z+e^{i \theta}\right)\right| d \theta
$$

imply that $g$ is bounded on $\mathbb{R}$. The Phragmèn-Lindelöf theorem yields the boundedness of $g(z)$ on every horizontal strip of finite width. Therefore, for each sequence $t_{m} \in \mathbb{R}$ there exists a subsequence $t_{m}^{\prime}$ such that the $f\left(z+t_{m}^{\prime}\right)$ converge uniformly to a function $h(z)$ on every compact subset of $\mathbb{C}$. If $h(z) \equiv 0$ on $\mathbb{C}$, then $\int_{0}^{2 \pi} \log \left|g\left(t_{m}^{\prime}+e^{i \theta}\right)\right| d \theta \rightarrow-\infty$. By (20), this contradicts (11); hence $g \in D$.

To prove the "only if" part, note that, by (6) , every $f \in C$ with $f(0) \neq 0$ has the form

$$
f(z)=A e^{i \lambda z} g(z)
$$

with $g$ as in (12). Conditions (7) and (8) follow immediately from (41) and (5). Using Lemma 1, we see that the zero set of $f$ satisfies condition (9) and, when $f \in B$, condition (10). If $f \in D$, then $f$ and $g$ are bounded on $\mathbb{R}$ and, by the Phragmèn-Lindelöf theorem, on every horizontal strip of finite width. Hence, if (11) is false, then, by (20),

$$
\frac{1}{2 \pi} \int_{0}^{2 \pi} \log \left|g\left(t_{l}+e^{i \theta}\right)\right| d \theta \rightarrow-\infty
$$

for some sequence $t_{j}$ of reals. By the properties of averages of subharmonic functions, we have

$$
\int_{\left|u+i v-t_{j}\right|<1} \log |g(u+i v)| d u d v \rightarrow-\infty .
$$

Since $\log |g|$ is bounded from above on the strip $|\operatorname{Im} z|<2$, we see that

$$
\int_{\left|u+i v-t_{j}-s\right|<2} \log |g(u+i v)| d u d v \rightarrow-\infty
$$

uniformly in $s \in[-1,1]$. Using the properties of averages of subharmonic functions once again, we obtain

$$
\sup _{-1 \leq s \leq 1} \log \left|g\left(t_{j}+s\right)\right| \rightarrow-\infty .
$$

On the other hand, $f\left(z+t_{j}^{\prime}\right) \rightarrow h(z) \not \equiv 0$ for some subsequence $t_{j}^{\prime}$ uniformly on the compact subsets of $\mathbb{C}$, and so (21) is impossible. Theorems 1-3 are proved completely.

Remark 1. It can be shown that all these theorems are valid with the function $\tilde{n}(c, t)=$ $\operatorname{card}\left\{a_{k}:\left|\operatorname{Re}\left(a_{k}-c\right)\right| \leq t,\left|\operatorname{Im}\left(a_{k}-c\right)\right| \leq t\right\}$ instead of $n(c, t)$ as well. 
Remark 2. The additional condition

$$
\limsup _{y \rightarrow \pm \infty} \int_{0}^{\infty}[n(b, t)-n(i y, t)](|y| t)^{-1} d t \leq \sigma
$$

in Theorems 1-3 gives a complete description of the zero sets for the corresponding subclasses of functions of exponential type at most $\sigma$.

Example. Let $\alpha(t)$ be a strictly monotone increasing concave function on $\mathbb{R}^{+}$with the properties $\alpha(0)=0,1 \leq \alpha^{\prime}(t) \leq 1+O\left(t^{-1}\right)$ as $t \rightarrow \infty$, and $\alpha(t) \geq t+1+\varepsilon$ for large $t$ with some $\varepsilon>0$. Consider a sequence of reals $\left\{a_{k}\right\}, k \in \mathbb{Z} \backslash\{0\}$, such that $\alpha\left(a_{k}\right)=k$ and $a_{-k}=-a_{k}, k \in \mathbb{N}$. Note that

$$
n(0, t)-n(x, t)=2 \mathrm{E}[\alpha(t)]+\mathrm{E}[\alpha(x-t)]-\mathrm{E}[\alpha(x+t)]
$$

for $x>t$, and

$$
n(0, t)-n(x, t)=2 \mathrm{E}[\alpha(t)]-E[\alpha(x-t)]-\mathrm{E}[\alpha(x+t)]
$$

for $0<x<t$; here $\mathrm{E}[x]$ is the integral part of $x$. Since $a_{n+1}-a_{n}=\left(\alpha^{\prime}(\tilde{t})\right)^{-1}$ with some $\tilde{t} \in\left(a_{n}, a_{n+1}\right)$, we see that

$$
0 \leq 1-\left(a_{n+1}-a_{n}\right) \leq\left(a_{n+1}-a_{n}\right)^{-1}-1 \leq \alpha^{\prime}\left(a_{n}\right)-1<C / a_{n}<1 / 2
$$

for $n \geq n_{0}$, and

$$
\left|\int_{a_{n}}^{a_{n+1}}(\alpha(t)-\mathrm{E}[\alpha(t)]-1 / 2) d t\right| \leq\left|\int_{a_{n}}^{a_{n+1}}\left(\int_{a_{n}}^{t} \alpha^{\prime}(u) d u-1 / 2\right) d t\right| \leq C^{\prime} / a_{n} .
$$

Therefore for any $N<\infty$ we have

$$
\left|\int_{a_{n_{0}}}^{a_{N}}(\alpha(t)-\mathrm{E}[\alpha(t)]-1 / 2) d t\right| \leq C^{\prime} \log a_{N} .
$$

Now it easily follows that all the integrals

$$
\begin{gathered}
\int_{1}^{x} \frac{\alpha(t)-\mathrm{E}[\alpha(t)]-1 / 2}{t} d t, \quad \int_{1}^{x} \frac{\alpha(x \pm t)-\mathrm{E}[\alpha(x \pm t)]-1 / 2}{t} d t \\
\int_{x}^{\infty} \frac{\alpha(t \pm x)-\mathrm{E}[\alpha(t \pm x)]-1 / 2}{t} d t
\end{gathered}
$$

are bounded uniformly in $x \rightarrow \infty$. Hence, up to a uniformly bounded term, the integral $\int_{0}^{\infty}[n(0, t)-n(x, t)] t^{-1} d t$ equals

$$
\begin{aligned}
\int_{1}^{x} \frac{2 \mathrm{E}[\alpha(t)]-2 t}{t} d t & +\int_{1}^{x-1} \frac{\alpha(x-t)-\alpha(x+t)+2 t}{t} d t \\
& +\int_{x}^{\infty} \frac{2 \alpha(t)-\alpha(t-x)-\alpha(x+t)}{t} d t
\end{aligned}
$$

Since $\alpha(t)$ is concave, the third integral in (22) is positive. Furthermore, for $1<t<x-1$ we have

$$
\alpha(x-t)-\alpha(x+t)+2 t=\int_{-t}^{t} 1-\alpha^{\prime}(x+u) d u \geq-C \log \frac{x+t}{x-t} .
$$

Now it is easy to check that the second integral in (22) is uniformly bounded from below. Obviously, the first integral in (22) is unbounded from above as $x \rightarrow \infty$. Thus, the sequence $\left\{a_{k}\right\}$ is not the zero set of any function $f \in B$. 


\section{REFERENCES}

[1] B. Ja. Levin, Distribution of zeros of entire functions, 'Gostekhizdat, Moscow, 1956; English transl., Transl. Math. Monogr., vol. 5, Amer. Math. Soc., Providence, RI, 1980. MR0087740 (19:402c) MR0589888 (81k:30011)

[2] , Lectures on entire functions, Transl. Math. Monogr., vol. 150, Amer. Math. Soc., Providence, RI, 1996. MR1400006 (97j:30001)

[3] P. Koosis, The logarithmic integral. I, Cambridge Stud. Adv. Math., vol. 12, Cambridge Univ. Press, Cambridge, 1988; II, Cambridge Stud. Adv. Math., vol. 21, Cambridge Univ. Press, Cambridge, 1992. MR0961844 (90a:30097) MR1195788 (94i:30027)

[4] B. N. Khabibullin, Completeness of systems of exponentials and sets of uniqueness, Bashkir. Univ., Ufa, 2006. (Russian)

Department of Mathematics, Kharkov National University, Svobody Square 4, Kharkov 61077, UKRAINE

E-mail address: favorov_s@mail.ru, fav@univer.kharkov.ua

Received 7/JUL/2006

Translated by THE AUTHOR 